\title{
On the impact of independence of irrelevant alternatives: the case of two-person NTU games*
}

\author{
Bezalel Peleg • Peter Sudhölter • José M. Zarzuelo
}

Received: 29 October 2010 / Accepted: 27 January 2011 / Published online: 24 February 2011

(C) The Author(s) 2011. This article is published with open access at SpringerLink.com

\begin{abstract}
On several classes of $n$-person NTU games that have at least one Shapley NTU value, Aumann characterized this solution by six axioms: Non-emptiness, efficiency, unanimity, scale covariance, conditional additivity, and independence of irrelevant alternatives (IIA). Each of the first five axioms is logically independent of the remaining axioms, and the logical independence of IIA is an open problem. We show that for $n=2$ the first five axioms already characterize the Shapley NTU value, provided that the class of games is not further restricted. Moreover, we present an example of a solution that satisfies the first five axioms and violates IIA for two-person NTU games $(N, V)$ with uniformly p-smooth $V(N)$.
\end{abstract}

\footnotetext{
*We are grateful to two anonymous referees of this journal for their comments that helped to improve the writing of this paper. The second and third author were supported by the Spanish Ministerio de Ciencia e Innovación under project ECO2009-11213, co-funded by the ERDF.
}

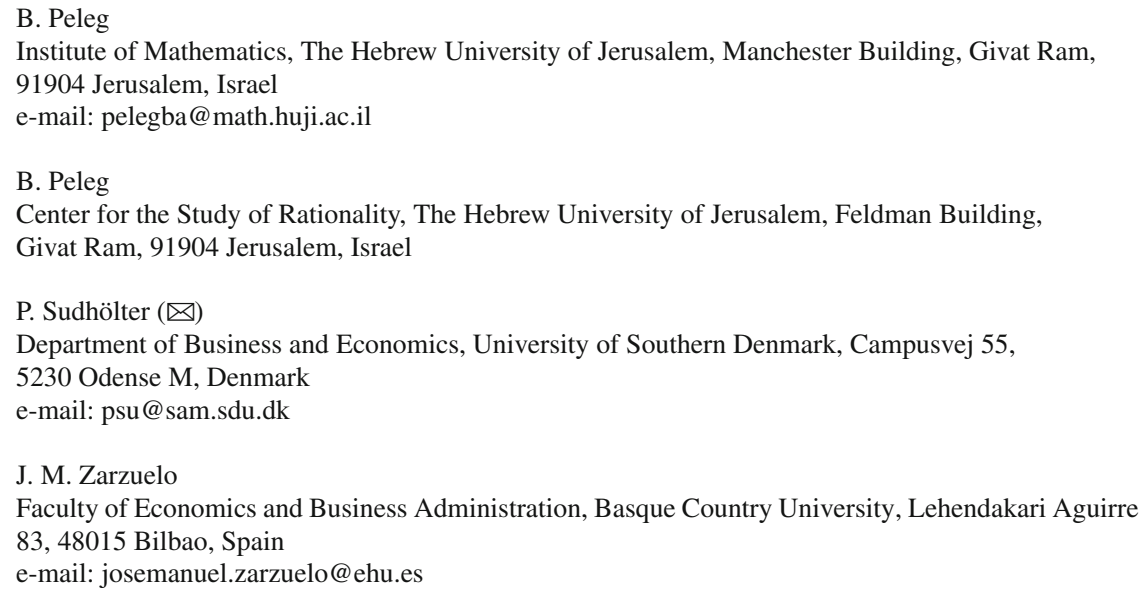


Keywords NTU game $\cdot$ Shapley NTU value $\cdot$ Positive smoothness

\author{
JEL Classification $\quad$ C71
}

\title{
1 Introduction
}

Several versions of Nash's (1950) axiom independence of irrelevant alternatives (IIA) have been employed and discussed in the literature in various fields of social sciences, e.g., Sen (1970) has used it under the name Property $\alpha$ in social choice in the connection with the theory of choice functions. In the context of NTU games, IIA (see Axiom 2 in Sect. 2 for a formal definition) requires that, quoting Aumann (1985), "a value $y$ of a game $W$ remains a value when one removes outcomes other than $y$ ("irrelevant alternatives') from the set $W(N)$ of all feasible outcomes, without changing $W(S)$ for coalitions other than the all player coalition." IIA is a natural generalization of one of the four properties-weak Pareto efficiency, equal treatment of equals, and scale covariance are the three others - in Nash's (1950) definition of the "Nash" solution for bargaining problems. The NTU value introduced by Shapley (1969), called "Shapley" NTU value, generalizes, on the one hand, the TU Shapley (1953) value and, on the other hand, the Nash solution for bargaining problems. According to Aumann, the Shapley NTU value is characterized by IIA and five further axioms whose TU versions characterize the TU Shapley value. Hart (1985) characterizes the Harsanyi NTU solution by suitably modified axioms. Thus, the open question ${ }^{1}$ whether IIA is really needed when NTU games are considered, is of particular interest. For the case of two-person games, we present a complete answer to the foregoing question in the following sense: On an interesting feasible class of two-person games IIA is logically independent of the remaining axioms, but if the class of games is rich enough, then the remaining axioms already imply IIA.

The paper is organized as follows. In Sect. 2 the basic notation is provided and those definitions and results due to Aumann (1985) that are relevant for our presentation are recalled, including his characterizations of the Shapley NTU value by six axioms, i.e., Theorem A and Theorem B.

Section 3 formulates our first main result: In the two-person case, IIA is not logically independent of the remaining axioms employed in Theorems A and B, if the considered class of games is rich enough. For uniformly p-smooth two-person games however, IIA is needed. The corresponding statement, Theorem 4.1, is our second main result, and Sect. 4 is devoted to the proof of this result. Finally, in Sect. 5 we discuss and show the logical independence of the remaining five axioms.

\footnotetext{
1 Indeed, using the symbol $\Lambda$ for the Shapley NTU value, Aumann (1985) writes in his Footnote 8: "A referee asked for an example to show that IIA is really needed, i.e., for a correspondence other than $\Lambda$ satisfying Axioms 0 through 5. We do not know of one. Thus at present, it is conceivable that Axioms 0 through 5 are already categoric." (His additional axiom of "Closure Invariance" is not relevant for NTU games with closed feasible sets.).
} 


\section{Some notation and preliminaries}

Let $N$ be a finite nonempty set. We denote by $\mathbb{R}^{N}$ the set of all real functions on $N$. So $\mathbb{R}^{N}$ is the $|N|$-dimensional Euclidean space. (Here and in the sequel, if $D$ is a finite set, then $|D|$ denotes the cardinality of $D$.) If $x, y \in \mathbb{R}^{N}$, then we write $x \geqslant y$ if $x_{i} \geqslant y_{i}$ for all $i \in N$. Moreover, we write $x>y$ if $x \geqslant y$ and $x \neq y$ and we write $x \gg y$ if $x_{i}>y_{i}$ for all $i \in N$. We denote $\mathbb{R}_{+}^{N}=\left\{x \in \mathbb{R}^{N} \mid x \geqslant 0\right\}$ and $\mathbb{R}_{++}^{N}=\left\{x \in \mathbb{R}^{N} \mid x \gg 0\right\}$. A coalition (in $N$ ) is a nonempty subset of $N$ and $2^{N}$ denotes the set of all subsets of $N$. For every $S \in 2^{N}$ and any $x, \lambda \in \mathbb{R}^{N}$, the indicator function on $S$ is denoted by $\chi^{S} \in \mathbb{R}^{N}$, i.e.,

$$
\chi_{j}^{S}= \begin{cases}1, & \text { if } j \in S, \\ 0, & \text { if } j \in N \backslash S,\end{cases}
$$

the scalar product $\sum_{i \in N} \lambda_{i} x_{i}$ is denoted by $\lambda \cdot x, \lambda * x=\left(\lambda_{i} x_{i}\right)_{i \in N}, \lambda_{S}$ is the restriction of $\lambda$ to $S$, and $0^{S}$ denotes the zero of $\mathbb{R}^{S}$, i.e., $0^{S}=0 \lambda_{S}$. For $A, B \subseteq \mathbb{R}^{N}, t \in \mathbb{R}$, we write $A+B=\{a+b \mid a \in A, b \in B\}, t A=\{t a \mid a \in A\}, \lambda * A=\{\lambda * a \mid a \in A\}$, and the boundary of $A, \operatorname{cl}(A) \cap \operatorname{cl}\left(\mathbb{R}^{N} \backslash A\right)$, is denoted by $\partial A$, where "cl" means "closure". If $A$ is convex and closed, then we say that $A$ is smooth if it has a unique supporting hyperplane at each $z \in \partial A$. We call $A$ comprehensive if $A=A-\mathbb{R}_{+}^{N}$.

A $T U$ game on $N$ is a mapping $v: 2^{N} \rightarrow \mathbb{R}$ with $v(\emptyset)=0$. An NTU game on $N$ is a mapping $V$ that assigns to each coalition $S$ in $N$ a nonempty comprehensive closed proper subset of $\mathbb{R}^{S}$ such that

(1) $V(N)$ is convex and smooth;

(2) $V(N)$ is non-leveled, i.e., if $x, y \in V(N)$ and $x>y$, then $y \notin \partial V(N)$;

(3) for each $S \in 2^{N} \backslash\{\emptyset, N\}$ there exits $x^{S} \in \mathbb{R}^{N}$ such that $V(S) \times\left\{0^{N \backslash S}\right\} \subseteq$ $V(N)+\left\{x^{S}\right\}$.

Moreover, we use the convention that $V(\emptyset)=\emptyset$. Let $\gamma_{N}$ and $\Gamma_{N}$ denote the set of all TU games and NTU games on $N$, respectively. For any $v \in \gamma_{N}$ the associated NTU game $V_{v} \in \Gamma_{N}$ is defined by $V_{v}(S)=\left\{y \in \mathbb{R}^{S} \mid y \cdot \chi_{S}^{S} \leqslant v(S)\right\}$ for all coalitions $S$ in $N$. Denote $\Gamma_{N}^{T U}=\left\{V_{v} \mid v \in \gamma_{N}\right\}$. For $T \in 2^{N} \backslash\{\emptyset\}$, the unanimity game on $T$, $u_{T} \in \gamma_{N}$, is defined by $u_{T}(S)=1$ for all $S$ such that $T \subseteq S \subseteq N$ and $u_{T}(S)=0$ for all $S \subseteq N$ with $T \backslash S \neq \emptyset$. The NTU unanimity game $U_{T}$ is the NTU game associated with $u_{T}$. The set $\gamma_{N}$ with coalition-wise operations is the real vector space of dimension $2^{|N|}-1$ and the set of TU unanimity games forms a basis of $\gamma_{N}$. Moreover, $\Gamma_{N}$ is closed under positive scalar multiplication, but, if $U, V \in \Gamma_{N}$, then $U+V$ may not be a member of $\Gamma_{N}$ provided $^{2}$ that $|N| \geqslant 2$. However, for any $\lambda \in \mathbb{R}_{++}^{N}$ and $V \in \Gamma_{N}, \lambda * V \in \Gamma_{N}$ (for any coalition $S,(\lambda * V)(S)=\lambda_{S} * V(S)$ ). One further notation is useful for the sequel. For any $V \in \Gamma_{N}$ let $d(V) \in \mathbb{R}^{N}$ be defined by

$$
d_{i}(V)=\max V(\{i\}) \text { for all } i \in N
$$

\footnotetext{
${ }^{2}$ If $U=U_{N}$ and $V=\lambda * U$ for some $\lambda \in \mathbb{R}_{++}^{N}$, then $U, V \in \Gamma_{N}$, but $U+V \notin \Gamma_{N}$ unless $\lambda_{i}=\lambda_{j}$ for all $i, j \in N$.
} 
Let $V \in \Gamma_{N}$. By (1) and comprehensiveness of $V(N)$, for any $x \in \partial V(N)$, there exists a unique $\lambda^{V, x} \in \mathbb{R}_{+}^{N}$ such that

$$
\chi^{N} \cdot \lambda^{V, x}=1 \text { and } V(N) \subseteq\left\{y \in \mathbb{R}^{N} \mid \lambda^{V, x} \cdot y \leqslant \lambda^{V, x} \cdot x\right\}
$$

Moreover, by (2), $\lambda^{V, x} \gg 0^{N}$ and, by (3), for any $S \in 2^{N}$,

$$
v_{x}^{V}(S)=\sup \left\{\lambda_{S}^{V, x} \cdot y \mid y \in V(S)\right\} \in \mathbb{R}
$$

with the convention that $v_{x}^{V}(\emptyset)=0$, so that $v_{x}^{V} \in \gamma_{N}$. Using this notation note that if $U, V, W=U+V \in \Gamma_{N}, x \in U(N), y \in V(N), \quad$ and $z=x+y \in \partial W(N)$, then $x \in \partial U(N), y \in \partial V(N), \lambda^{U, x}=\lambda^{V, y}=\lambda^{W, z}, \quad$ and $v_{x}^{U}+v_{y}^{V}=v_{z}^{W}$.

Now, the Shapley NTU value (the NTU value for short) of $V$ introduced by Shapley (1969), denoted by $\Phi(V)$, is defined by

$$
\Phi(V)=\left\{x \in \partial V(N) \mid \lambda^{V, x} * x=\phi\left(v_{x}^{V}\right)\right\},
$$

where, for any $v \in \gamma_{N}$, the Shapley value [see Shapley (1953)] of $v$, denoted by $\phi(v) \in \mathbb{R}^{N}$, is defined by

$$
\phi_{i}(v)=\sum_{S \subseteq N \backslash\{i\}} \frac{|S| !(|N|-|S|-1) !}{|N| !}(v(S \cup\{i\})-v(S)) \text { for all } i \in N \text {. }
$$

Let $\Gamma \subseteq \Gamma_{N}$. A solution on $\Gamma$ is a mapping $\sigma$ that assigns to each $V \in \Gamma$ a subset $\sigma(V)$ of $V(N)$. The following properties of a solution $\sigma$ on $\Gamma \subseteq \Gamma_{N}$ are employed.

- Axiom 1 (Non-Emptiness, NE): $\sigma(V) \neq \emptyset$ for all $V \in \Gamma$.

- Axiom 2 (Efficiency, EFF): $\sigma(V) \subseteq \partial V(N)$ for all $V \in \Gamma$.

- Axiom 3 (Conditional Additivity, CADD): If $U, V, W=U+V \in \Gamma$, then $\sigma(W) \supseteq$ $(\sigma(U)+\sigma(V)) \cap \partial W(N)$.

- Axiom 4 (Unanimity, UNA): If $U_{T} \in \Gamma$, then $\sigma\left(U_{T}\right)=\left\{\frac{\chi^{T}}{|T|}\right\}$ for $T \in 2^{N} \backslash\{\emptyset\}$.

- Axiom 5 (Scale Covariance, SCOV): If $V \in \Gamma, \lambda \in \mathbb{R}_{++}^{N}$, and $\lambda * V \in \Gamma$, then $\sigma(\lambda * V)=$ $\lambda * \sigma(V)$.

- Axiom 6 (Independence of Irrelevant Alternatives, IIA): If $U, V \in \Gamma, U(N) \subseteq$ $V(N)$, and $U(S)=V(S)$ for all $S \varsubsetneqq N$, then $\sigma(U) \supseteq \sigma(V) \cap U(N)$.

In order to recall Aumann's characterization of $\Phi$, the following definition is useful.

Definition 2.1 Let $N$ be a finite nonempty set and $\Gamma \subseteq \Gamma_{N}$. Then $\Gamma$ is a feasible domain if

(1) $\Phi(V) \neq \emptyset$ for all $V \in \Gamma$;

(2) $\Gamma_{N}^{T U} \subseteq \Gamma$; 
(3) If $V \in \Gamma$ and $\lambda \in \mathbb{R}_{++}^{N}$, then $\lambda * V \in \Gamma$;

(4) If $V \in \Gamma$, then the game that is obtained by replacing $V(N)$ by any of its supporting half-spaces is an element of $\Gamma$, i.e., if $x \in \partial V(N)$, and if $W \in \Gamma_{N}$ is the game that may differ from $V$ only inasmuch as $W(N)=\left\{y \in \mathbb{R}^{N} \mid \lambda^{V, x} \cdot y \leqslant \lambda^{V, x} \cdot x\right\}$, then $W \in \Gamma$.

Let $N$ be a finite nonempty set. We remark that $\Gamma_{N}^{\Phi}=\left\{V \in \Gamma_{N} \mid \Phi(V) \neq \emptyset\right\}$ is a feasible domain.

Theorem 2.2 (Aumann (1985, Theorem A)) Let $\Gamma \subseteq \Gamma_{N}$ be a feasible domain. Then the Shapley NTU value is the unique solution on $\Gamma$ that satisfies Axioms 1 through 6.

Axiom 6, the IIA axiom, in the foregoing theorem may be replaced by "maximality":

Theorem 2.3 (Aumann (1985, Theorem B)) Let $\Gamma \subseteq \Gamma_{N}$ be a feasible domain. Then the Shapley NTU value is the maximum solution on $\Gamma$ that satisfies Axioms 1 through 5; i.e., $\Phi$ satisfies Axioms 1 through 5 on $\Gamma$, and if the solution $\sigma$ on $\Gamma$ satisfies Axioms 1 through 5, then $\sigma(V) \subseteq \Phi(V)$ for all $V \in \Gamma$.

\section{The class of two-person games with a Shapley value}

The main result of this section is the following theorem.

Theorem 3.1 If $|N|=2$, then the Shapley NTU value on $\Gamma_{N}^{\Phi}$ is characterized by Axioms 1 through 5.

We postpone the proof and present several preparatory remarks and lemmas. Throughout this section, let $|N|=2$, say $N=\{1,2\}$.

Remark 3.2 Let $V \in \Gamma_{N}$. If $d(V) \in V(N)$, then $|\Phi(V)|=1$. If $d(V) \in \partial V(N)$, then $\Phi(V)=\{d(V)\}$.

For any function $g: \mathbb{R} \rightarrow \mathbb{R} \cup\{-\infty\}$ let $\operatorname{dom}(g)=\{x \in \mathbb{R} \mid g(x) \in \mathbb{R}\}$, i.e., $\operatorname{dom}(g)$ denotes the effective domain of $g$. We say that $g: \mathbb{R} \rightarrow \mathbb{R} \cup\{-\infty\}$ is differentiable if $g^{\prime}(x)$ exists for any $x \in \operatorname{dom}(g)$. Let

$$
\begin{aligned}
\Gamma^{0} & =\left\{V \in \Gamma_{N} \mid d(V)=0\right\} \text { and } \\
\mathcal{G} & =\{g: \mathbb{R} \rightarrow \mathbb{R} \cup\{-\infty\} \mid g \text { is concave and differentiable, } \operatorname{dom}(g) \\
& \left.\neq \emptyset, g^{\prime}(x)<0 \text { for all } x \in \operatorname{dom}(g)\right\} .
\end{aligned}
$$

Note that, for any $g \in \mathcal{G}$, by concavity of $g$, the derivative of $g$ on $\operatorname{dom}(g)$ is continuous.

The mapping that assigns to each $V \in \Gamma^{0}$ the function $g_{V}: \mathbb{R} \rightarrow \mathbb{R} \cup\{-\infty\}$ defined by

$$
g_{V}(x)=\sup \{y \in \mathbb{R} \mid(x, y) \in V(N)\},
$$

where $\sup \emptyset=-\infty$, is a bijection from $\Gamma^{0}$ to $\mathcal{G}$. Hence, for each $V \in \Gamma^{0}$,

$$
\left\{\left(x, g_{V}(x)\right) \mid x \in \operatorname{dom}\left(g_{V}\right)\right\}=\partial V(N) .
$$


Let $V \in \Gamma^{0}$ and $g=g_{V}$. It is well-known (see, e.g., Maschler et al. (1988, (1))) that

$$
(x, y) \in \Phi(V) \Leftrightarrow x \in \operatorname{dom}(g), \quad y=g(x), g^{\prime}(x) x=-g(x) .
$$

It is useful to use another parametrization of $\partial V(N)$. Substituting any $(x, g(x)), x \in$ $\operatorname{dom}(g)$, by $(t-f(t),-t-f(t))$ yields $g^{\prime}(x)\left(1-f^{\prime}(t)\right)=-1-f^{\prime}(t)$ so that

$$
f^{\prime}(t)=\frac{-1-g^{\prime}(x)}{1-g^{\prime}(x)}
$$

and hence $-1<f^{\prime}(t)<1$ and $f: \mathbb{R} \rightarrow \mathbb{R}$ is convex. We have deduced that the mapping that assigns to each $V \in \Gamma^{0}$ the convex differentiable function $f_{V}:=f$ is a bijection from $\Gamma^{0}$ to $\mathcal{F}$, where

$$
\mathcal{F}=\left\{f: \mathbb{R} \rightarrow \mathbb{R} \mid f \text { is a convex } C^{1} \text { function, }-1<f^{\prime}(t)<1 \text { for all } t \in \mathbb{R}\right\} .
$$

Lemma 3.3 Let $V \in \Gamma^{0}$. For all $t \in \mathbb{R},\left(t-f_{V}(t),-t-f_{V}(t)\right) \in \Phi(V)$ iff $t=f_{V}(t)$ $f_{V}^{\prime}(t)$.

Proof Let $f=f_{V}, g=g_{V}, x \in \operatorname{dom}(g)$, and $t=\frac{x-g(x)}{2}$. By (3.2),

$$
f(t) f^{\prime}(t)-t=\left(\frac{-x-g(x)}{2}\right)\left(\frac{-1-g^{\prime}(x)}{1-g^{\prime}(x)}\right)-\frac{x-g(x)}{2}=\frac{g^{\prime}(x) x+g(x)}{1-g^{\prime}(x)} .
$$

We conclude that $f(t) f^{\prime}(t)=t$ if and only if $g^{\prime}(x) x=-g(x)$. The proof is complete by (3.1).

Corollary 3.4 Let $U^{0} \in \Gamma^{0}$ satisfy

$$
\begin{aligned}
f_{U^{0}}(0) & >0, \\
f_{U^{0}}(t) f_{U^{0}}(t) & >t \text { for all } t>0, \\
f_{U^{0}}(t) f_{U^{0}}(t) & <t \quad \text { for all } t<0 .
\end{aligned}
$$

Then, for any $U \in \Gamma^{0}$ that satisfies

$$
\begin{aligned}
& f_{U}(0)=f_{U^{0}}(0), \\
& f_{U}^{\prime}(t) \geqslant f_{U^{0}}(t) \quad \text { for all } t>0, \\
& f_{U^{\prime}}(t) \leqslant f_{U^{0}}(t) \quad \text { for all } t<0
\end{aligned}
$$

the following two properties are satisfied:

$$
\begin{aligned}
f_{U}^{\prime}(\mathbb{R}) & =]-1,1[ \\
\Phi(U) & =\left\{\left(-f_{U}(0),-f_{U}(0)\right)\right\} .
\end{aligned}
$$


Proof In order to show (3.9), by (3.7) and (3.8), it suffices to verify that $\sup _{q \in \mathbb{R}} f_{U^{0}}{ }^{\prime}(q)=1$ and that $\inf _{q \in \mathbb{R}} f_{U^{0}}{ }^{\prime}(q)=-1$. However, by (3.4) and (3.5), $f_{U^{0}}(t)>t$ for all $t>0$ and $f_{U^{0}}(t)<t$ for all $t<0$ so that the foregoing equations are implied by

$f_{U^{0}}(t) \leqslant t \sup _{q \in \mathbb{R}} f_{U^{0^{\prime}}}(q)+f_{U^{0}}(0) \quad$ and $\quad f_{U^{0}}(t) \geqslant t \inf _{q \in \mathbb{R}} f_{U^{0}}(q)+f_{U^{0}}(0) \quad$ for all $t \in \mathbb{R}$.

By (3.3) - (3.8), $t=f_{U}(t) f_{U}^{\prime}(t)$ iff $t=0$. Thus, (3.10) follows from Lemma 3.3.

We now construct, for any $\alpha>0$, a game $U^{0} \in \Gamma^{0}$ that satisfies (3.4), (3.5), and $f_{U^{0}}(0)=\alpha$. Secondly, a useful technical Lemma is proved.

For $\varepsilon>0$ and $c \in \mathbb{R}$, let $V^{\varepsilon, c} \in \Gamma^{0}$ be defined by

$$
V^{\varepsilon, c}(N)=\left\{x \in \mathbb{R}^{N} \mid x_{1}<0, x_{1} x_{2} \geqslant \varepsilon^{2}\right\}-\left\{c \chi^{N}\right\} .
$$

Remark 3.5 It is straightforward to verify that, for any $t \in \mathbb{R}$,

$$
f_{V^{\varepsilon, 0}}(t)=\sqrt{t^{2}+\varepsilon^{2}}
$$

so that, by Lemma 3.3, $\Phi\left(V^{\varepsilon, 0}\right)=\partial V^{\varepsilon, 0}(N)$. By Definition of $V^{\varepsilon, c}$, for any $c \in \mathbb{R}$, $f_{V^{\varepsilon, c}}(t)=f_{V^{\varepsilon, 0}}(t)+c$. Again by Lemma 3.3, $\{(c, c)\}=\Phi\left(V^{\varepsilon, c}\right)$ for all $c \in \mathbb{R} \backslash\{0\}$. Furthermore, for any $c>0, U^{0}=V^{\varepsilon, c}$ satisfies (3.3) $-(3.5)$ and $f_{U^{0}}(0)=\varepsilon+c$.

Lemma 3.6 Let $g, h: \mathbb{R}_{+} \rightarrow \mathbb{R}_{+}$be continuous and nondecreasing functions such that $g(0)=h(0)=0$ and $g(t) \leqslant h(t)$ for all $t \in \mathbb{R}_{+}$. Then there exist continuous and nondecreasing functions $\widetilde{h}, s: \mathbb{R}_{+} \rightarrow \mathbb{R}_{+}$such that

$$
\begin{aligned}
& \widetilde{h}(0)=0, \widetilde{h}(t) \geqslant h(t) \quad \text { for all } t \in \mathbb{R}_{+}, \\
& \widetilde{h}\left(\mathbb{R}_{+}\right)=h\left(\mathbb{R}_{+}\right), \\
& \widetilde{h}(s(t))=g(t) \quad \text { for all } t \in \mathbb{R}_{+}, \\
& s(0)=0 \leqslant s(t)-s\left(t^{\prime}\right) \leqslant t-t^{\prime} \quad \text { for all } t, t^{\prime} \in \mathbb{R}_{+}, t^{\prime} \leqslant t .
\end{aligned}
$$

Proof In order to construct $\widetilde{h}: \mathbb{R}_{+} \rightarrow \mathbb{R}$, we introduce, for any $q \in \mathbb{R}_{+}$, the auxiliary function $g_{q}: \mathbb{R}_{+} \rightarrow \mathbb{R}$ defined by $g_{q}(t)=g(t+q)$ for all $t \geqslant 0$. Moreover, let $f: \mathbb{R}_{+} \rightarrow \mathbb{R}_{+} \cup\{\infty\}$ be defined by $f(q)=\inf \left\{t \in \mathbb{R}_{+} \mid g_{q}(t)=h(t)\right\}$ for all $q \geqslant 0$ (with the convention that inf $\emptyset=\infty$ ). Note that "inf" is in fact "min", because $g$ and $h$ are continuous. Now, define

$$
\widetilde{h}(t)=\sup \left(\{h(t)\} \cup\left\{g_{q}(t) \mid q \geqslant 0, f(q) \leqslant t\right\}\right) \text { for all } t \in \mathbb{R}_{+} .
$$

By construction, $\widetilde{h}$ is nondecreasing and satisfies (3.13).

Let $t \in \mathbb{R}_{+}$. If there exists $q$ with $f(q) \leqslant t$ and $g_{q}(t)>h(t)$, then $\left\{q \in \mathbb{R}_{+} \mid f(q) \leqslant t\right\}$ is a compact interval so that "sup" is, in fact, "max" in any case. Consequently, the continuities of $h$ and $g$ imply the continuity of $\widetilde{h}$ and, hence, (3.14). 
For $\gamma \in g\left(\mathbb{R}_{+}\right)$denote

$$
\begin{array}{ll}
\alpha_{g}(\gamma)=\min \left\{t \in \mathbb{R}_{+} \mid g(t)=\gamma\right\}, & \alpha_{\widetilde{h}}(\gamma)=\min \left\{t \in \mathbb{R}_{+} \mid \widetilde{h}(t)=\gamma\right\}, \\
\beta_{g}(\gamma)=\sup \left\{t \in \mathbb{R}_{+} \mid g(t)=\gamma\right\}, & \beta_{\widetilde{h}}(\gamma)=\sup \left\{t \in \mathbb{R}_{+} \mid \widetilde{h}(t)=\gamma\right\} .
\end{array}
$$

We may now define $s: \mathbb{R}_{+} \rightarrow \mathbb{R}_{+}$as follows. For $t \geqslant 0$ let $s(t)=$ $\min \left\{\alpha_{\widetilde{h}}(\gamma)+t-\alpha_{g}(\gamma), \beta_{\widetilde{h}}(\gamma)\right\}$, where $\gamma=g(t)$. By construction, $s$ is nondecreasing and satisfies (3.15). As $\beta_{\widetilde{h}}(\gamma)-\alpha_{\widetilde{h}}(\gamma) \leqslant \beta_{g}(\gamma)-\alpha_{g}(\gamma)$ (note that $\beta_{g}(\gamma)=\infty$ is just possible if $\max _{t} g(t)$ exists and $\left.\gamma=\max _{t} g(t)\right), s$ is continuous, and it satisfies (3.16).

Now, we are prepared for the proof.

Proof of Theorem 3.1: By Aumann's Theorem B we only have to show uniqueness. Let $\sigma$ be a solution on $\Gamma_{N}^{\Phi}$ that satisfies NE, PO, CADD, UNA, and SCOV, let $V \in \Gamma_{N}^{\Phi}$. Again by Theorem B, $\sigma(V) \subseteq \Phi(V)$ so that it suffices to prove that $\Phi(V) \subseteq \sigma(V)$. If $\Phi(V)$ is a singleton, then the proof is finished by NE. Hence, by Remark 3.2 we may assume that $d \notin V(N)$, where $d=d(V)$. Let $\widehat{x} \in \Phi(V)$. It remains to show that $\widehat{x} \in \sigma(V)$. By SCOV we may assume that $\widehat{x}=d-2 \chi^{N}$.

By CADD and Remark 3.2 it suffices to construct $U, W \in \Gamma_{N}^{\Phi}$ such that $\Phi(U)=$ $\left\{-2 \chi^{N}\right\}, d=d(W) \in \partial W(N)$, and $V=U+W$.

In order to construct $U$, an auxiliary game $U^{1} \in \Gamma_{N}^{\Phi}$ is constructed. Let $U^{1}$ be the NTU game defined by $U^{1}(N)=\frac{1}{2}(V(N)-\{d\})-\left\{\chi^{N}\right\}$ and $d\left(U^{1}\right)=0$. Then $U^{1} \in \Gamma_{N}^{\Phi}$ and $-2 \chi^{N} \in \Phi\left(U^{1}\right)$. By Remark 3.5 there exists $U^{0} \in \Gamma^{0}$ that satisfies $(3.3)-(3.5)$ and $f_{U^{0}}(0)=2$. Let $f_{i}=f_{U^{i}}$ for $i=0,1$. Recall that $\left.f_{0}^{\prime}(\mathbb{R})=\right]-1,1[$. Let $\widetilde{F}: \mathbb{R} \rightarrow$ ] $-1,1[$ be any continuous and strictly increasing function that satisfies

$$
\widetilde{F}(t) \begin{cases}\geqslant \max _{i \in\{0,1\}} f_{i}^{\prime}(t), & \text { if } t \geqslant 0, \\ \leqslant \min _{i \in\{0,1\}} f_{i}^{\prime}(t), & \text { if } t<0 .\end{cases}
$$

By the aforementioned properties of the functions $\left.f_{i}^{\prime}, \widetilde{F}(\mathbb{R})=\right]-1,1[$ and $\widetilde{F}(0)=0$.

Applying Lemma 3.6 to $g, h: \mathbb{R}_{ \pm}$given by $g(t)=f_{1}^{\prime}(t)$ and $h(t)=\widetilde{F}(t)$ (or given by $g(t)=-f_{1}^{\prime}(-t)$ and $h(t)=-\widetilde{F}(-t)$, respectively), for all $t \geqslant 0$, guarantees the existence of continuous nondecreasing functions $F: \mathbb{R} \rightarrow]-1,1[$ and $s: \mathbb{R} \rightarrow \mathbb{R}$ that satisfy

$$
\begin{aligned}
& F(t) \geqslant \widetilde{F}(t), F(-t) \leqslant \widetilde{F}(-t) \text { for all } t \in \mathbb{R}_{+}, \\
& F(s(t))=f_{1}^{\prime}(t) \quad \text { for all } t \in \mathbb{R}_{+}, \\
& s(0)=0 \leqslant s(t)-s\left(t^{\prime}\right) \leqslant t-t^{\prime} \text { for all } t, t^{\prime} \in \mathbb{R}, t^{\prime} \leqslant t .
\end{aligned}
$$

Let $f: \mathbb{R} \rightarrow \mathbb{R}$ be the unique function defined by $f^{\prime}=F$ and $f(0)=2$. Then $f$ is a convex $\mathrm{C}^{1}$ function. Let $U$ be the zero-normalized NTU game defined by

$$
U(N)=\left\{x \in \mathbb{R}^{N} \mid x \leqslant(t-f(t),-t-f(t)) \text { for some } t \in \mathbb{R}\right\} .
$$


As $f^{\prime}(t) \geqslant f_{0}^{\prime}(t)$ for all $t>0$ and $f^{\prime}(t) \leqslant f_{0}^{\prime}(t)$ for all $t<0, \Phi(U)=\left\{-2 \chi^{N}\right\}$ by Corollary 3.4 so that $U \in \Gamma^{0}$.

By (3.19), the real function $\widehat{s}: \mathbb{R} \rightarrow \mathbb{R}$ defined by $\widehat{s}(t)=2 t-s(t)$ for all $t \in \mathbb{R}$ is a monotonic continuous bijection that satisfies $\widehat{s}(0)=0$. Hence there exists a unique $\mathrm{C}^{1}$ function $g$ that satisfies $g(0)=2$ and $g^{\prime}(t)=f_{1}^{\prime}\left(\widehat{s}^{-1}(t)\right)$. Then $g$ is convex, $g^{\prime}(0)=0$, and $\left.g^{\prime}(t) \in\right]-1,1[$ so that the NTU game $W$ defined by $W(\{i\})=V(\{i\})$ for $i \in N$ and

$$
W(N)=\left\{x \in \mathbb{R}^{N} \mid x \leqslant(t-g(t),-t-g(t)) \text { for some } t \in \mathbb{R}\right\}+\left\{2 \chi^{N}+d\right\}
$$

satisfies (1) and (2) of Sect. 2. As $d=d(W) \in \partial W(N), \Phi(W)=\{d\}$ by Remark 3.2.

Let $h=f \circ s+g \circ \widehat{s}$. We claim that

$$
h^{\prime}(t)=2 f_{1}^{\prime}(t) \text { for all } t \in \mathbb{R}
$$

In order to show (3.20) define $D_{f}, D_{g}: \mathbb{R}^{2} \rightarrow \mathbb{R}$ by

$$
D_{f}\left(t, t^{\prime}\right)=\left\{\begin{array}{cl}
\frac{f(t)-f\left(t^{\prime}\right),}{t-t^{\prime}}, & \text { if } t \neq t^{\prime}, \\
f^{\prime}(t), & \text { if } t=t^{\prime},
\end{array} \text { and } \quad D_{g}\left(t, t^{\prime}\right)=\left\{\begin{array}{cl}
\frac{g(t)-g\left(t^{\prime}\right)}{t-t^{\prime}}, & \text { if } t \neq t^{\prime} \\
g^{\prime}(t), & \text { if } t=t^{\prime}
\end{array}\right.\right.
$$

and note that $D_{f}, D_{g}$ are continuous. Hence, for any $t \in \mathbb{R}$,

$$
\begin{aligned}
h^{\prime}(t) & =\lim _{t^{\prime} \rightarrow t} \frac{f(s(t))-f\left(s\left(t^{\prime}\right)\right)+g(\widehat{s}(t))-g\left(\widehat{s}\left(t^{\prime}\right)\right)}{t-t^{\prime}} \\
& =\lim _{t^{\prime} \rightarrow t} \frac{D_{f}\left(s(t), s\left(t^{\prime}\right)\right)\left(s(t)-s\left(t^{\prime}\right)\right)+D_{g}\left(\widehat{s}(t), \widehat{s}\left(t^{\prime}\right)\right)\left(\widehat{s}(t)-\widehat{s}\left(t^{\prime}\right)\right)}{t-t^{\prime}} \\
& =\lim _{t^{\prime} \rightarrow t} \frac{\left(D_{f}\left(s(t), s\left(t^{\prime}\right)\right)-D_{g}\left(\widehat{s}(t), \widehat{s}\left(t^{\prime}\right)\right)\right)\left(s(t)-s\left(t^{\prime}\right)\right)+D_{g}\left(\widehat{s}(t), \widehat{s}\left(t^{\prime}\right)\right)\left(2 t-2 t^{\prime}\right)}{t-t^{\prime}}
\end{aligned}
$$

As $f^{\prime}(s(t))=f_{1}^{\prime}(t)=g^{\prime}(\widehat{s}(t))$ for all $t \in \mathbb{R}$, we may conclude from (3.19) and the continuities of $s$ and $\widehat{s}$ that

$$
\begin{gathered}
\lim _{t^{\prime} \rightarrow t} \frac{\left(D_{f}\left(s(t), s\left(t^{\prime}\right)\right)-D_{g}\left(\widehat{s}(t), \widehat{s}\left(t^{\prime}\right)\right)\right)\left(s(t)-s\left(t^{\prime}\right)\right)}{t-t^{\prime}}=0, \\
\lim _{t^{\prime} \rightarrow t} \frac{D_{g}\left(\widehat{s}(t), \widehat{s}\left(t^{\prime}\right)\right)\left(2 t-2 t^{\prime}\right)}{t-t^{\prime}}=2 g^{\prime}(\widehat{s}(t))
\end{gathered}
$$

so that our claim follows.

Now, $h(0)=4=2 f_{1}(0)$ so that $h=2 f_{1}$. By definition of $f_{1}$,

$$
U^{1}(N)=\left\{x \in \mathbb{R}^{N} \mid x \leqslant\left(t-f_{1}(t),-t-f_{1}(t)\right) \text { for some } t \in \mathbb{R}\right\}
$$


so that

$$
\begin{aligned}
\partial V & (N)-\left\{d+2 \chi^{N}\right\} \\
& =2 \partial U^{1}(N) \\
& =\left\{\left(2 t-2 f_{1}(t),-2 t-2 f_{1}(t)\right) \mid t \in \mathbb{R}\right\} \\
& =\{(2 t-h(t),-2 t-h(t)) \mid t \in \mathbb{R}\} \\
& =\{(s(t)-f(s(t))+\widehat{s}(t)-g(\widehat{s}(t)),-s(t)-f(s(t))-\widehat{s}(t)-g(\widehat{s}(t))) \mid t \in \mathbb{R}\}
\end{aligned}
$$

so that $V(N) \subseteq U(N)+W(N)$ is shown. In order to show that $U(N)+W(N) \subseteq V(N)$, as $U(N)+W(N) \subseteq\{d\}+\left\{x \in \mathbb{R}^{N} \mid x(N) \leqslant-4\right\}$, it suffices to show that any element of $\partial(U(N)+W(N))$ belongs to $V(N)$. Let $x \in \partial(U(N)+W(N))$. Then there exist $y \in \partial U(N)$ and $z \in \partial W(N)$ such that $x=y+z$. Let $t, \alpha \in \mathbb{R}$ such that $x-d-2 \chi^{N}=(t-\alpha,-t-\alpha)$. By the definition of $U$ and $W$ there exist $t^{\prime}, t^{\prime \prime} \in \mathbb{R}$ such that $y=\left(t^{\prime}-f\left(t^{\prime}\right),-t^{\prime}-f\left(t^{\prime}\right)\right)$ and $z-d-2 \chi^{N}=\left(t^{\prime \prime}-g\left(t^{\prime \prime}\right),-t^{\prime \prime}-g\left(t^{\prime \prime}\right)\right)$. As the supporting hyperplane to $U(N)$ at $y$ is parallel to the supporting hyperplane to $W(N)$ at $z$ [see (2.4], $f^{\prime}\left(t^{\prime}\right)=g^{\prime}\left(t^{\prime \prime}\right)$. As $s(t / 2)+\widehat{s}(t / 2)=t$, there exists $\beta \in \mathbb{R}$ such that $t^{\prime}=s(t / 2)+\beta$ and $t^{\prime \prime}=\widehat{s}(t / 2)-\beta$. As $f^{\prime}(s(t / 2))=g^{\prime}(\widehat{s}(t / 2))$ and $f^{\prime}$ and $g^{\prime}$ are nondecreasing functions, $f^{\prime}\left(t^{\prime}\right)=f^{\prime}(s(t / 2))=g^{\prime}\left(t^{\prime \prime}\right)$ and $\alpha=2 f_{1}(t / 2)$.

Remark 3.7 As pointed out by one anonymous referee, Theorem 3.1 may be attacked on the grounds that the definition of the Shapley NTU value is used in the characterization in the following sense: Each of the considered games must have at least one Shapley NTU value and Axiom 1 requires non-emptiness of the solution. However, it should be remarked that, for $|N|=2$, say $N=\{1,2\}$, and $V \in \Gamma_{N}$ there is a necessary and sufficient condition for non-emptiness of $\Phi(V)$ : If $d(V) \in V(N)$, then $V \in \Gamma_{N}^{\Phi}$ (see Remark 3.2). If $d(V) \notin V(N)$, then $V \in \Gamma_{N}^{\Phi}$ if and only if there exists $y \ll d(V)$ such that $y \in \partial V(N)$ and $\lambda^{V, y}=\lambda^{W, y}$, where $W$ is the NTU game such that $d(W)=d(V)=d$ and $\partial W(N)$ is the hyperbola

$$
\left\{z \in \mathbb{R}^{N} \mid z \ll d(W),\left(z_{1}-d_{1}\right)\left(z_{2}-d_{2}\right)=\left(y_{1}-d_{1}\right)\left(y_{2}-d_{2}\right)\right\} .
$$

Indeed, $\Phi(W)=\partial W(N)$ by Remark 3.5.

\section{The class of uniformly p-smooth two-person games}

In order to state the main theorem of this section, the following definition is needed. Let $N$ be a finite nonempty set and let $V \in \Gamma_{N}$. Then $V$ is called uniformly p-smooth if there exists $\varepsilon>0$ such that $\lambda^{V, x} \geqslant \varepsilon \chi^{N}$ for all $x \in \partial V(N)$ (for the definition of $\lambda^{V, x}$ see (2.2)). Note that Maschler and Owen (1992) introduced uniform p-smoothness in order to receive a quite general existence result for their "consistent Shapley value".

Theorem 4.1 If $|N|=2$ and $\Gamma \subseteq \Gamma_{N}$ is the set of uniformly p-smooth NTU games, then $\Gamma$ is a feasible domain and Axiom 6 (IIA) is logically independent of the remaining axioms in Theorem A. 
This section is devoted to the proof of Theorem 4.1 by means of an example of an appropriate subsolution of the Shapley NTU value.

Throughout this section, let $|N|=2$, say $N=\{1,2\}$, and let $\Gamma^{\text {ups }}$ denote the set of uniformly p-smooth games in $\Gamma_{N}$. Clearly, $\Gamma^{\text {ups }}$ satisfies (2) - (4) of Definition 2.1. In order to show that $\Gamma^{u p s}$ is a feasible domain in $\Gamma_{N}$, it suffices to construct, for any $V \in \Gamma^{u p s}$, a nonempty subset of $\Phi(V)$. To this end let $V \in \Gamma^{u p s}$ and define

$$
\sigma_{0}(V)= \begin{cases}\Phi(V), & \text { if } d(V) \in V(N), \\ \arg \max \left\{\left(d_{1}(V)-x_{1}\right)\left(d_{2}(V)-x_{2}\right) \mid x \in \partial V(N)\right\}, & \text { if } d(V) \notin V(N) .\end{cases}
$$

Note that $\sigma_{0}$ is well-defined. Indeed, if $d(V) \notin V(N)$, then $\partial V(N) \cap\left(\{d(V)\}-\mathbb{R}_{+}^{N}\right)$ is a nonempty compact set by uniform p-smoothness of $V(N)$ so that $\sup \left\{\left(d_{1}(V)-x_{1}\right)\left(d_{2}(V)-x_{2}\right) \mid x \in V(N)\right\}$ is attained by some $x \in \partial V(N), x \ll$ $d(V)$.

By Remark 3.2, $\sigma_{0}$ satisfies NE. Moreover, it satisfies SCOV and UNA. In order to show that $\sigma_{0}(V) \subseteq \Phi(V)$, we may assume that $d(V) \notin V(N)$. Let $x \in \sigma_{0}(V)$, $t=\left(d_{1}(V)-x_{1}\right)\left(d_{2}(V)-x_{2}\right)$, and $\lambda=\lambda^{V, x}$ (see (2.2)). Then the hyperplane $\left\{z \in \mathbb{R}^{N} \mid \lambda \cdot z=\lambda \cdot x\right\}$ is a tangent to the hyperbola

$$
\left\{z \in \mathbb{R}^{N} \mid z \ll d(V),\left(d_{1}(V)-z_{1}\right)\left(d_{2}(V)-z_{2}\right)=t\right\}
$$

so that $x \in \Phi(V)$ by (3.1) and the well-known translation covariance of $\Phi$.

We now show that $\sigma_{0}$ satisfies CADD.

\section{Lemma 4.2 The solution $\sigma_{0}$ on $\Gamma^{u p s}$ satisfies CADD.}

Proof For $i \in\{1,2\}$, let $V^{i} \in \Gamma^{u p s}, x^{i} \in \sigma_{0}\left(V^{i}\right)$ such that, with $V=V^{1}+V^{2}$ and $x=x^{1}+x^{2}, V \in \Gamma^{u p s}$ and $x \in \partial V$. By CADD of $\Phi, x \in \Phi(V)$. It remains to show that $x \in \sigma_{0}(V)$. If $d=d(V) \in V(N)$, then the proof is finished. Hence, we may assume that $d / \in V(N)$. As $x \in \partial V(N), \lambda^{V^{i}, x^{i}}=\lambda^{V, x}$ for $i=1,2$, by (2.4). By (2.5), there exists $c \in \mathbb{R}$ such that $\left(d^{2}-x^{2}\right)=c\left(d^{1}-x^{1}\right)$, where $d^{i}=d\left(V^{i}\right)$ for $i=1,2$. As $d=d^{1}+d^{2}$, Remark 3.2 implies that $x^{1} \ll d^{1}$ or $x^{2} \ll d^{2}$. Without loss of generality we may assume that $x^{1} \ll d^{1}$. By definition of $\sigma_{0}$,

$$
V^{1}(N) \supseteq\left\{z \in \mathbb{R}^{N} \mid z \ll d^{1},\left(d_{1}^{1}-z_{1}\right)\left(d_{2}^{1}-z_{2}\right) \geqslant\left(d_{1}^{1}-x_{1}^{1}\right)\left(d_{2}^{1}-x_{2}^{1}\right)\right\}=: Z^{1}
$$

Let $Z=\left\{z \in \mathbb{R}^{N} \mid z \ll d, \prod_{i \in N}\left(d_{i}-z_{i}\right) \geqslant \prod_{i \in N}\left(d_{i}-x_{i}\right)\right\}$. Two cases may occur:

(1) $x^{2} \geqslant d^{2}$. By (4.1), $V(N) \supseteq\left\{x^{2}\right\}+Z^{1}$. Let $z \in Z$ and define $z^{1}=z-x^{2}$. It suffices to show that $z^{1} \in Z^{1}$. Now, $z^{1} \ll d^{1}$, because $x^{2} \geqslant d^{2}$ and $z \ll d$. The statement immediately follows from:

$$
\begin{aligned}
a, b \in \mathbb{R}_{++}^{N}, a_{1} a_{2} & \geqslant b_{1} b_{2}, \alpha \geqslant 0 \Longrightarrow\left(a_{1}+\alpha b_{1}\right)\left(a_{2}+\alpha b_{2}\right) \\
& \geqslant\left(b_{1}+\alpha b_{1}\right)\left(b_{2}+\alpha b_{2}\right)=(1+\alpha)^{2} b_{1} b_{2} .
\end{aligned}
$$


In order to show (4.2) we may assume that $a_{1} a_{2}=b_{1} b_{2}$, i.e., $a_{2}=b_{1} b_{2} / a_{1}$. Define $f\left(a_{1}\right)=\left(a_{1}+\alpha b_{1}\right)\left(\frac{b_{1} b_{1}}{a_{1}}+\alpha b_{2}\right)$. Then $f$ is a convex function and $f^{\prime}\left(a_{1}\right)=0$ iff $a_{1}=b_{1}$.

(2) $x^{2} \ll d^{2}$. Let

$$
Z^{2}=\left\{z \in \mathbb{R}^{N} \mid z \ll d^{2},\left(d_{1}^{2}-z_{1}\right)\left(d_{2}^{2}-z_{2}\right) \geqslant\left(d_{1}^{2}-x_{1}^{2}\right)\left(d_{2}^{2}-x_{2}^{2}\right)\right\} .
$$

By definition of $\sigma_{0}, V^{2}(N) \supseteq Z^{2}$. As $Z^{1}+Z^{2} \supseteq Z$, the proof is finished.

Example 4.3 shows that $\sigma_{0} \neq \Phi$.

Example 4.3 Let $X=\left\{x \in \mathbb{R}^{N} \mid x \ll 0, x_{1} x_{2}=1\right\}$ and $U \in \Gamma_{N}$ be defined by $U(N)=X-\mathbb{R}_{+}^{N}$ and $d(V)=0^{N}$. If $Y=\left\{y \in X \mid x_{i} \geqslant-3\right\}$, then $Y \neq \emptyset$ so that

$$
W(N):=\left\{z \in \mathbb{R}^{N} \mid \lambda^{U, y} \cdot z \leqslant \lambda^{U, y} \cdot y \text { for all } y \in Y\right\}
$$

is uniformly p-smooth. Let $d(W)=0^{N}$. We may easily deduce that $\Phi(W)=Y$. Let $d=\chi^{N}$ and $V \in \Gamma^{u p s}$ be defined by $V(N)=W(N)$ and $d(V)=\chi^{N}$. By symmetry of $V, \Phi(V) \ni-d$. Define $x$ by $x_{1}=-3$ and $x_{2}=-\frac{1}{3}$ and observe that $x \in \partial V(N)$. However, $\left(d_{1}-x_{1}\right)\left(d_{2}-x_{2}\right)=16 / 3>4$ so that $-d \notin \sigma_{0}(V)$.

\section{The logical independence of the remaining axioms}

The following examples show that even in the case $|N|=2$ each of the Axioms 1 through 5 are logically independent of the remaining axioms in Theorem A and in Theorem B. As far as we know, the logical independence of these axioms was only checked for Theorem A and the case $|N| \geqslant 3$ (see Peleg and Sudhölter (2007)) so that, in particular, the solution $\sigma_{1}$ defined below reveals some additional insight in the proof of Theorem B.

Throughout this section, let $N$ be a finite set such that $|N| \geqslant 2$. Let $\Gamma \subseteq \Gamma_{N}$ be a feasible domain. We are now going to define, for $i=1, \ldots, 5$, a solution $\sigma_{i}$ on $\Gamma$ that exclusively violates Axiom $i$ in Theorem $\mathrm{A}$ as well as in Theorem $\mathrm{B}$, even if "maximum" is replaced by "unique maximal". 3

In order to define $\sigma_{1}$, note that, as mentioned in Sect. 2, any TU game $v$ on $N$ is a linear combination of unanimity games, that is, there exist unique $c_{T}(v)=c_{T}, \varnothing \neq$ $T \subseteq N$, such that $v=\sum_{\emptyset \neq T \subseteq N} c_{T} u_{T}$. As $|N| \geqslant 2$, there exist $2^{|N|}-1 \geqslant 3$ coalitions. Select any two distinct coalitions $T^{1}$ and $T^{2}$ and define $\gamma_{N}^{+}=\left\{v \in \gamma_{N} \mid c_{T^{1}}(v), c_{T^{2}}(v) \geqslant 0\right\}$ and $\gamma_{N}^{++}=\left\{v \in \gamma_{N} \mid c_{T^{1}}(v), c_{T^{2}}(v)>0\right\}$.

\footnotetext{
3 A solution $\sigma$ is the unique maximal solution that satisfies certain axioms, if (a) $\sigma$ satisfies the axioms, (b) $\sigma$ is maximal under (a) (i.e., any solution that satisfies the axioms and contains $\sigma$ coincides with $\sigma$ ), and (c) there exists no further maximal solution that satisfies the axioms.
} 
For any $V \in \Gamma$ define

$$
\sigma_{1}(V)=\left\{x \in \partial(V) \mid v_{x}^{V} \in \gamma_{N}^{++}\right\} \cup\left\{x \in \Phi(V) \mid v_{x}^{V} \in \gamma_{N}^{+}\right\}
$$

Clearly, $\sigma_{1}$ satisfies EFF, SCOV, and IIA. As any unanimity TU game is an element of $\gamma_{N}^{+} \backslash \gamma_{N}^{++}, \sigma_{1}$ satisfies UNA. CADD follows from (2.4). As $\sigma_{1}\left(V_{-u_{N}}\right)=\emptyset, \sigma_{1} \neq \Phi$. Regarding the aforementioned modification of Theorem B, it remains to show that $\sigma_{1}$ is a maximal solution that satisfies the remaining axioms, i.e., Axioms 2 through 5. Assume, on the contrary, there exists a solution $\sigma$ that satisfies EFF, CADD, UNA, SCOV, and contains $\sigma_{1}$ as a proper subsolution. Let $V \in \Gamma$ such that there exists $x \in \sigma(V) \backslash \sigma_{1}(V)$. By EFF, $x \in \partial V(N)$. Let $v=v_{x}^{V}, \lambda=\lambda^{V, x}, \widehat{\lambda}=\left(1 / \lambda_{i}\right)_{i \in N}$, and $c_{T}=c_{T}(v)$ for $T \in 2^{N} \backslash\{\emptyset\}$. Let $W$ be the NTU game associated with

$$
w=\sum_{R \in 2^{N} \backslash\left\{\emptyset, T^{2}\right\}}\left(-c_{R}\right) u_{R}+\left(1+\left|c_{T^{2}}\right|\right) u_{T^{2}} .
$$

Two cases may occur:

(1) $c_{T^{1}}<0$ or $c_{T^{2}}<0$, say $c_{T^{1}}<0$. Then $w \in \gamma_{N}^{++}$so that $\partial(\widehat{\lambda} * W)(N)=\sigma_{1}(\widehat{\lambda} * W) \subseteq$ $\sigma(\widehat{\lambda} * W)$. Now, $V+\widehat{\lambda} * W=\widehat{\lambda} *\left(1+\left|c_{T^{2}}\right|\right) U_{T^{2}}$ so that, by $\operatorname{SCOV}, \sigma(V+\widehat{\lambda} * W)$ is a singleton. On the other hand, by CADD, $\partial(V+\widehat{\lambda} * W)(N) \subseteq \sigma(V+\widehat{\lambda} * W)$ so that the desired contradiction has been obtained.

(2) $c_{T^{1}}, c_{T^{2}} \geqslant 0, c_{T^{1}} c_{T^{2}}=0$, and $\lambda * x \neq \phi(v)$, say $c_{T^{1}}=0$. Then $V+\widehat{\lambda} * W=$ $\widehat{\lambda} *\left(1+c_{T^{2}}\right) U_{T^{2}}$ so that, By SCOV and UNA, $\sigma(V+\widehat{\lambda} * W)=\Phi(V+\widehat{\lambda} * W)$. As $w \in \gamma_{N}^{+} \backslash \gamma_{N}^{++}$in this case, $\sigma(\widehat{\lambda} * W)=\Phi(\widehat{\lambda} * W)$ so that CADD, applied to $x$ and the unique element of $\sigma(\widehat{\lambda} * W)$ yields the desired contradiction.

In order to define the solution $\sigma_{2}$ that exclusively violates EFF and contains $\Phi$ as a subsolution, we distinguish two cases: If $|N|>2$, then let $\sigma_{2}$ be the solution defined by Peleg and Sudhölter (2007, Sect. 13.3, p 242), denoted by $\sigma^{2}$. If $|N|=2$, then define

$$
\sigma_{2}(V)= \begin{cases}\Phi(V), & \text { if } d(V) \notin V(N) \text { or } d(V)=0 \\ \Phi(V) \cup\{d(V)\}, & \text { otherwise. }\end{cases}
$$

Clearly, $\sigma_{2}$ satisfies NE and SCOV, and it violates EFF. By (2.4), $\sigma_{2}$ inherits CADD from $\Phi$. Moreover, UNA and IIA are easily deduced using Remark 3.2.

The straightforward proofs that, for an arbitrary $|N| \geqslant 2$, the following solutions satisfy the desired properties, are left to the reader.

$$
\begin{aligned}
& \sigma_{3}(V)=\Phi(V) \cup\{x \in \partial V(N) \mid x \ll d(V)\} ; \\
& \sigma_{4}(V)=\partial V(N) ; \\
& \sigma_{5}(V)=\Phi(V) \cup\left\{x \in \partial V(N) \mid \lambda^{V, x} \neq \frac{\chi^{N}}{|N|}\right\} .
\end{aligned}
$$


Open Access This article is distributed under the terms of the Creative Commons Attribution License which permits any use, distribution and reproduction in any medium, provided the original author(s) and source are credited.

\section{References}

Aumann RJ (1985) An axiomatization of the non-transferability utility value. Econometrica 53:599-612

Hart S (1985) An axiomatization of Harsanyi's non-transferable utility solution. Econometrica 53: 1445-1450

Maschler M, Owen G (1992) The consistent Shapley value for games without side payments. In: Selten R (ed) Rational interaction: essays in honor of John Harsanyi, pp 5-12, New York. Springer-Verlag

Maschler M, Owen G, Peleg B (1988) Paths leading to the Nash set. In: Roth AE (ed) The Shapley value: essays in honor of Lloyd S. Shapley, pp 321-330, Cambridge. Cambridge University Press

Nash JF (1950) The bargaining problem. Econometrica 18:155-162

Peleg B, Sudhölter P (2007) Introduction to the Theory of Cooperative Games. Theory and Decision Library, Series C, 2nd edn. Springer-Verlag, Berlin

Sen AK (1970) Collective Choice and Social Welfare. Holden-Day, San Francisco, distribution taken over by North-Holland, Amsterdam

Shapley LS (1953) A value for $n$-person games. in Contribution to the Theory of Games II, vol 28, Annals of Mathematics Studies, Princeton, N.J. Princeton University Press, pp 307-317

Shapley LS (1969) Utility comparisons and the theory of games. in La Decision, aggregation et dynamique des ordres de preference, Paris. Edition du Centre National de le Recherche Scientifique, pp 251-263 